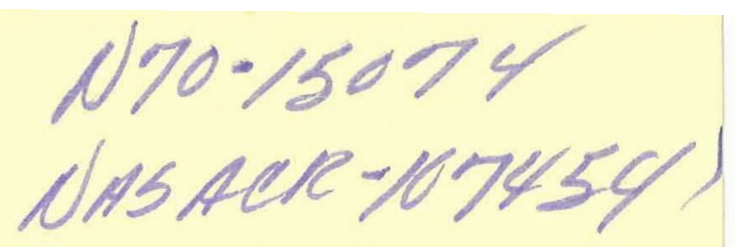

\title{
A SHORT, CYLINDRICAL ANTENNA AS A DIAGNOSTIC PROBE FOR MEASURING COLLISION FREQUENCIES IN A COLLISION-DOMINATED, NON-MAXWELLIAN PLASMA
}

By

L.D. Scott and B. Rama Rao

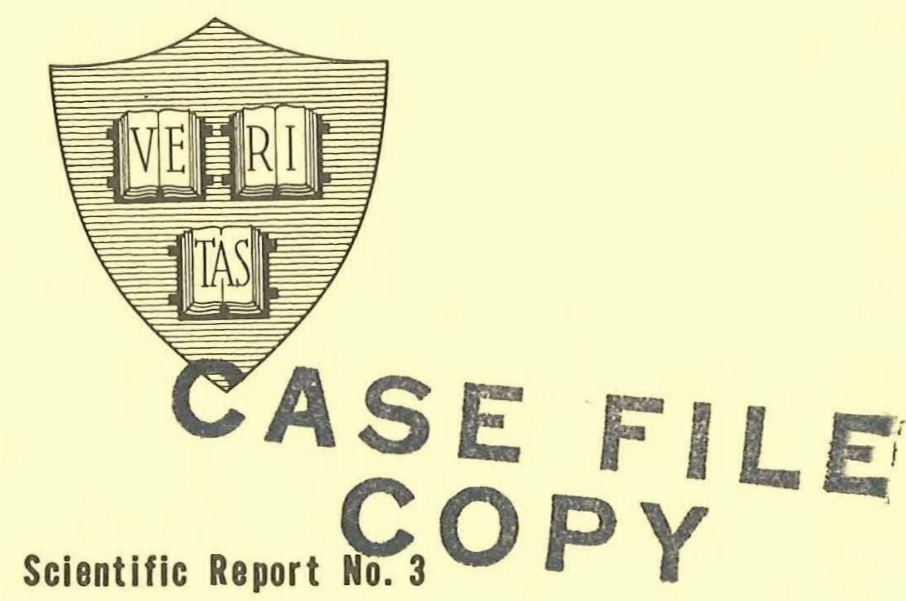

March 1969

"Reproduction in whole or in part is permitted by the U. S.

Government. Distribution of this document is unlimited."

MATIONAL AEROMAUTICS AND SPACE ADMINISTRATION

Prepared under Grant NGR 22-007-056

Division of Engineering and Applied Physics

Harvard University - Cambridge, Massachusetts 
A SHORT, CYLINDRICAL ANTENNA AS A DIAGNOSTIC PROBE FOR MEASURING COLLISION FREQUENCIES IN A COLLISION-DOMINATED, NON-MAXWELLIAN PLASMA

By

L. D. Scott and B. Rama Rao

Scientific Report No. 3

Reproduction in whole or in part is permitted by the U. S. Government. Distribution of this document is unlimited.

March 1969

Prepared under Grant NGR 22-007-056

Division of Engineering and Applied Physics

Harvard University, Cambridge, Massachusetts

for

NATIONAL AERONAUTICS AND SPACE ADMINISTRATION 


\title{
A SHORT, CYLINDRICAL ANTENNA AS A DIAGNOSTIC PROBE FOR MEASURING COLLISION FREQUENCIES IN A COLLISION-DOMINATED, NON-MAXWELLIAN PLASMA
}

\author{
By \\ L. D. Scott and B. Rama Rao \\ Gordon McKay Laboratory \\ Harvard University \\ Cambridge, Massachusetts 02138
}

\begin{abstract}
Investigations have been made to determine the effects of inter particle collisions on the antiresonant impedance characteristics of an electrically short, cylindrical antenna in the vicinity of the plasma fre. quency of an isotropic non-Maxwellian plasma. The dependence of the electron-neutral collision frequency on the electron energy has been taken into account. The experimental results have been compared with the theories proposed by King et al. ${ }^{1}$ and Balmain. ${ }^{2}$ The use of this antenna as a diagnostic probe for measuring electron-neutral collision frequency and electron density has been investigated. Electron-neutral collision frequencies for Helium measured by this technique are in good agreement with theoretical results calculated from the collision crosssection data of Golden and Bandel. 3
\end{abstract}




\section{Introduction}

Although electrostatic Langmuir probes ${ }^{4}$ and $r, f$, resonance probes 5 have been used for a number of years for making reliable measurements of the electron density and electron temperature of plasmas, no satisfactory r.f. probe technique appears to have been developed as yet, for measuring electron-neutral collision frequencies of either laboratory or ionospheric plasmas. Typical radio frequency diagnostic methods used for measuring collision frequencies of plasmas include a wide variety of $r$. $f$ attenuation measurements ${ }^{6,7}$ or some form of a cross modulation technique ${ }^{8}$.

The primary purpose of this paper is to investigate the impedance behavior of a short, cylindrical antenna in the vicinity of the plasma frequency of a weakly-ionized, is otropic, collision-dominated, non-Maxwellian plasma. Secondly, it is of considerable interest to explore the possibility of using such an antenna as a diagnostic probe for measuring electronneutral collision frequencies. Since the antenna is electrically very short, the values of the collision frequency and electron density are averaged over the 'near-field' region of the antenna, the dimensions of which are quite small, so that it becomes possible to get information on the local characteristics of the plasma. Missile antennas of this type have been used by Haycock, Baker, and Ulwick ${ }^{9}$ and by Jackson and Kane ${ }^{10}$ for electron density measurements, by measuring the change in the antenna reactance at the plasma frequency. They have, however, not extended their technique for measuring collision frequencies by considering the change in the input resistance of the antenna. The quasi-static theories used in their calculations also leave much to be desired since the effect of the antenna dimensions and plasma parameters have not been properly accounted for. Mlodnosky 
and Garriott ${ }^{11}$ have used a dipole antenna for measuring electron density and electron temperature in a loss-less plasma; their method consists of measuring the effect of the ion-sheath on the antenna admittance at frequencies much below the plasma frequency. In contrast to these earlier investigations, this paper is devoted mainly to making accurate measurements of collision frequency and electron density in a collision-dominated plasma because, under these conditions, the contribution to the antenna impedance from the electron temperature and ion-sheath effects can be neglected. The probe is, therefore, likely to be useful for diagnostic measurements in high pressure, rare-gas laboratory-discharge plasmas, shock-tube plasmas, and some types of re-entry plasmas, and also in the D-region of the ionosphere ${ }^{7}$, provided that the effects of the earth's magnetic field are properly accounted for. ${ }^{12}$

The two principal difficulties encountered in applying such a technique for collision frequency measurements are 1) the lack of an accurate theory which takes into account the effect of the various plasma parameters such as the electron density, collision frequency, electron temperature, and non-collisional damping on the input impedance of the antenna, and 2) the discrepancy in the expression for the plasma conductivity obtained from the Lorentzian model (which is commonly used for determining antenna impedance) and the kinetic theory model derived from the Boltzmann equation. ${ }^{13}$ The following two sections of this paper will elaborate on these topics. 
2. Theory for the Input Impedance of a Short, Cylindrical Antenna in

Cold and Warm Plasmas.

Numerous theoretical papers ${ }^{14}$ have been published in recent years describing the impedance and radiation characteristics of antennas immersed in plasmas. As the literature on this problem is very vast only those papers which are most relevant to this investigation are mentioned here. The experimental results, for the most part, have been analyzed using the theory proposed by King, Harrison and Denton ${ }^{1}$ for an antenna in a cold, lossy, isotropic plasma. In order to estimate the contribution from the electroacoustic: mode to the antenna impedance in the vicinity of the plasma frequency, the results have also been compared with the theory proposed by Balmain ${ }^{2}$ for a warm plasma based on a linearized, hydrodynamic model. More recently, Galejs ${ }^{15}$ has treated a similar problem using a variational technique; unfortunately, this paper came to our attention too late to permit numerical calculations for comparison. Numerical solutions of the integral equations for cylindrical antennas immersed in warm plasmas have been made by Wunsch, ${ }^{16}$ Lin and Mei, ${ }^{17}$ Cook and Edgar, ${ }^{18}$ and by Kuehl. ${ }^{19}$ These authors, however, have ignored collisional effects and, hence, their theory is not applicable to our experiments.

In the theory outlined by King, Harrison and Denton, the plasma is treated as a Lorentzian gas with a collisional damping term that is assumed to be independent of the electron velocity. The current distribution on the antenna is solved by an integral equation technique and the input admittance $Y(k)$ of the antenna is given by the following equations: 


$$
Y_{\text {in }}(k)=G_{i n}(k)+j B_{i n}(k)
$$

where

$$
G_{i n}(k)=\frac{2 \pi}{\zeta_{e} \psi_{d l}}\left\{\frac{2 \alpha}{\beta}\left[\beta h+\frac{2}{3} \beta^{3} h^{3} F\left(1-\frac{\alpha^{2}}{\beta^{2}}\right)\right]+\frac{\beta^{4} h^{4}}{3(\Omega-3)}\left(1-\frac{10 \alpha^{2}}{\beta^{2}}+\frac{5 \alpha^{4}}{\beta^{4}}\right)\right\}
$$

and

$B_{\text {in }}(k)=\frac{2 \pi}{\zeta_{e} \psi_{d l}}\left\{\beta h\left(1-\frac{\alpha^{2}}{\beta^{2}}\right)+\frac{1}{3} \beta^{3} h^{3} F\left(1-\frac{6 \alpha^{2}}{\beta^{2}}+\frac{\alpha^{4}}{\beta^{4}}\right)-\frac{\beta^{4} h^{4}}{3(\Omega-3)} \frac{\alpha}{\beta}\left(5-\frac{10 \alpha^{2}}{\beta^{2}}+\frac{\alpha^{4}}{\beta^{4}}\right)\right.$

In the above equation $G_{\text {in }}(k)$ and $B_{\text {in }}(k)$ are, respectively, the input conductance and input susceptance of the antenna; $2 \mathrm{~h}$ and a are the length and radius of the cylindrical antenna; $F=1+[(3 \ln (2)-1) /(\Omega-3)]$, and $\Omega=2 \ln (2 \mathrm{~h} / \mathrm{a})$.

$$
\alpha=\omega \sqrt{\mu \mid \varepsilon_{\mathrm{e}}} \mathrm{f}(\mathrm{p}) \text { and } \beta=\omega \sqrt{\mu \mid \varepsilon_{\mathrm{e}}} \mathrm{g}(\mathrm{p}) \text { when } \mathrm{p}<0 \text { and } \epsilon<0 .
$$

and

$$
\alpha=\omega \sqrt{\mu \epsilon_{\mathrm{e}}} g(p) \text { and } \beta=\omega \sqrt{\mu \varepsilon_{\mathrm{e}}} \mathrm{f}(\mathrm{p}) \text { when } \mathrm{p}>0 \text { and } \varepsilon>0 \text { 。 }
$$

where

$$
f(p)=\sqrt{\frac{1}{2} \sqrt{1+p^{2}}+1}
$$

and

$$
g(p)=\sqrt{\frac{1}{2} \sqrt{1+p^{2}}-1}
$$

In equations $(1-3), \zeta_{\mathrm{e}}=\omega \mu / \beta, \psi_{\mathrm{dl}}=2 \ln (\mathrm{h} / \mathrm{a})-2, \mathrm{p}=\sigma_{\mathrm{e}} / \omega \epsilon_{\mathrm{e}}$ where 


$$
\sigma_{e}=\frac{\epsilon_{0} \omega_{p}^{2} \nu_{c}}{\nu_{c}^{2}+\omega^{2}} \text { and } \epsilon_{e}=\epsilon_{0}\left[1-\frac{\omega_{p}^{2}}{\omega^{2}+\nu_{c}^{2}}\right]
$$

$\omega_{\mathrm{p}}, \nu_{\mathrm{c}}$ and $\omega$ are, respectively, the angular plasma frequency, collision frequency, and signal frequency of the antenna.

The input admittance of the antenna given by equations (1-3) has been computed for a wide range of plasma parameters corresponding to the experimental conditions and has been compared with the measured results.

The theory by King et al. does not take into account the electron temperature which causes a coupling of energy between the electromagnetic and electroacoustic modes, particularly near the plasma frequency. In order to estimate the relative contribution by electroacoustic and collisional effects, computations of the antenna impedance were also made from the theory proposed by Balmain ${ }^{2}$ for an isotropic, compressible plasma; the formula for the antenna impedance $Z$ obtained from Poynting's theorem with an assumed triangular current distribution on the antenna is given by ( $R$ ef, 2, Eq, 30)

$$
Z_{\text {in }}=Z_{E M}+Z_{P}
$$

where

$$
Z_{E M}=\frac{1}{j \omega \pi \epsilon_{0} k_{0} h}[\ln (h / a)-1]
$$

and

$$
\begin{aligned}
& Z_{P}=\frac{1}{j \omega \pi \epsilon_{0} k_{0} h}\left\{\left(1-k_{0}\right) \frac{\pi}{2}\left[J_{0}(\tau a) N_{0}(\tau a)+j J_{0}^{2}(\tau a)\right]\right\} \quad \text { when } \omega>\omega_{P} \\
& Z_{P}=\frac{-1}{j \omega \pi \epsilon_{0} k_{0} h}\left[\left(1-k_{0}\right) I_{0}(\rho a) K_{0}(\rho a)\right] \quad \text { when } \omega<\omega_{p}
\end{aligned}
$$


In equation $(4 \mathrm{c}), \rho=j \tau$ and

$\rho^{2}=\frac{\omega^{2}(X-U)}{v^{2}}, X=\frac{\omega_{p}^{2}}{\omega^{2}}, \quad \omega_{p}^{2}=\frac{n e^{2}}{m \varepsilon_{0}}, U=1-j Z, Z=\frac{\nu_{c}}{\omega}, \quad k_{0}=1-\frac{X}{U}$

$v=\sqrt{\frac{3 k T}{m}}$ is the acoustical velocity in the electron gas. In equations (4a) and $(4 b) Z_{E M}$ and $Z_{P}$ are the contributions from the electromagnetic and electroacoustic modes to the input impedance of the antenna. Equation (4c) shows that even for a lossless plasma, the antenna has a resistive component owing to the contribution from the plasma mode when $\omega>\omega_{\mathrm{p}^{\circ}}$ It is also seen from (4b) that the resistive part $R_{\text {em }}$ of the electromagnetic mode impedance is zero when the plasma has no losses; this result is a consequence of the quasi-static approximation made in the analys is. When small collisional losses are introduced, $\mathrm{R}_{\mathrm{em}}$ is a maximum near the plasma frequency. The values of $R_{e m}$ and $R_{p}$ for typical experimental electrontemperatures and collision frequencies have been calculated and their relative magnitudes have been compared. These results will be discussed later in this paper.

\section{Electrical Conductivity of a Non-Maxwellian Plasma.}

In deriving an expression for the antenna impedance, King et al. ${ }^{1}$ have assumed the plasma to be a 'Lorentzian' gas, where the collision term is independent of the electron energy; the a.c. electrical conductivity of the plasma obtained from Langevin's equation is given by

$$
\sigma=\frac{n e^{2}}{m} \frac{(\nu-j \omega)}{m\left(\nu_{e}^{2}+\omega^{2}\right)}
$$


where $\nu_{e}$ is the effective collision frequency. Although Balmain has considered the electroacoustic radiation due to the finite electron temperature of the plasma, his results are valid only for a Maxwellian plasma since he has assumed that the collision frequency is independent of electron temperature; hence, his expression for plasma conductivity for the electromagnetic mode is identical to the one used by King.

Although this simple 'corventional' expression for the conductivity has been used quite extensively in the literature, especially by ionospheric physicists, it is still subject to criticism since it is not strictly applicable to a 'statistical' ensemble of electrons having a distribution of energy, but, rather, to a 'microcanonical' assembly with a known, average electron energy. To obtain a more rigorous expression for the plasma conductivity based on statistical considerations one needs to start with the Boltzmann equation; a solution has been obtained by Allis ${ }^{20}$ and Margenau ${ }^{21}$ by expanding the distribution function in terms of $s$ pherical harmonics in velocity space and by Fourier series in time. The high frequency electronic conductivity of the plasma is given by 22

$$
\sigma=\frac{n e^{2}}{m}[B-j D]
$$

where

$$
B=\frac{4 \pi}{3} \int_{0}^{\infty} f_{1} v^{4} d v
$$

and

$$
D=-\frac{4 \pi}{3} \int_{0}^{\infty} \frac{\omega}{\nu_{m}} f_{1} v^{4} d v
$$

and

$$
f_{1}=-\frac{v_{m}}{v_{m}^{2}+\omega^{2}} \frac{1}{v} \frac{d F_{0}^{0}}{d v}
$$


where $\nu_{m}$ is the electron-neutral velocity-dependent collision frequency for momentum transfer. $F_{0}^{0}$ is the symmetrical part of the distribution function and depends only on the velocity $\vec{v}$. It is the distribution for electrons prevailing in the plasma when the $r . f$ field on the antenna is absent. In most cases it is reasonable to assume that this distribution is Maxwellian in nature, with anarbitrary electron temperature $\mathrm{T}_{\mathrm{e}}$.

$$
F_{0}^{0}=(\beta / \pi)^{3 / 2} e^{-\beta v^{2}}
$$

where

$$
\beta=\mathrm{m} / 2 \mathrm{kT}
$$

The collision frequency of momentum transfer $\nu_{m}$ within the integral sign in $(6 b-d)$ can be expressed as

$$
v_{m}=\rho Q v
$$

where $\rho$ is the number of gas atoms per cubic centimeter.

$$
\rho=2.687 \times 10^{19}(\mathrm{P} / 760)\left(273 / \mathrm{T}_{\mathrm{g}}\right)
$$

where $P$ is the neutral gas pressure in Torr, $T_{g}$ is the neutral gas temperature in degrees Kelvin. $Q$ is the electron-neutral collision cross-section for momentum transfer. Substitution of (7) and (8) into (6b) yields

$$
f_{1}=\frac{\rho Q v 2 \beta(\beta / \pi)^{3 / 2} \exp \left(-\beta v^{2}\right)}{\rho^{2} Q^{2} v^{2}+\omega^{2}}
$$

For a Maxwellian gas, the cross-section for momentum transfer $Q$ varies inversely as the electron velocity. Hence, $\nu_{\mathrm{m}}$ is a constant with respect to electron velocity. Thus, from equation (6b) 


$$
B=\frac{4 \pi}{3} \frac{\nu_{m}}{\nu_{m}^{2}+\omega^{2}} 2 \beta(\beta / \pi)^{3 / 2} \int_{0}^{\infty} \exp \left(-\beta v^{2}\right) v^{4} d v
$$

The integral on the right hand side may be evaluated by using the identity

$$
\int_{0}^{\infty} e^{-\beta v^{2}} v^{4} d v=\frac{3}{8}\left(\sqrt{\pi} / \beta^{5 / 2}\right)
$$

From equations $(6),(10)$ and (11) it follows that for a Maxwellian gas

$$
\sigma=\frac{n e^{2}}{m}\left[\frac{\nu_{m}-j \omega}{v_{m}^{2}+\omega^{2}}\right]
$$

A comparison of equations (5) and (12) indicates that for a Maxwellian gas the effective collision frequency $\nu_{e}$ obtained from the Lorentzian model is equal to the average collision frequency $\nu_{m}(v)$ where $\bar{v}=\left(3 \mathrm{kT}_{\mathrm{v}} / \mathrm{m}\right)^{1 / 2}$ is the root mean square velocity.

However, most gaseous plasmas are non-Maxwellian in nature; the collision frequency of these gases has a strong functional dependency on the electron velocity. For example, for air and nitrogen $Q$ is proportional to velocity. For water $Q$ varies as $1 / V^{2}$. In the experiments described. in this paper, the gas used for collision frequency measurements was Helium. The collision cross-section $Q$ of Helium as a function of electron energy is shown in Figure 5 of Reference 3 . It can be seen from this figure that ionized Helium is a non-Maxwellian plasma since the $Q$ is reasonably constant with respect to electron velocity. Hence, the effective collision frequency $\nu_{e}$ cannot be simply equated with the collision frequency 
obtained from kinetic theory as defined in equation (6). Numerical and experimental comparisons of the two conductivity expressions for nonMaxwellian plasmas such as air, etc, have been made by Margenau and Stillinger ${ }^{23}$ and by Kane ${ }^{24}$; large discrepancies between the two models have been noticed. It follows that in any experiment involving a precise measurement of the collision frequency using electromagnetic methods, it is necessary to discriminate between the approximate and accurate formula for the conductivity.

Several authors have suggested convenient mathematical expedients in order to relate the two models and to define the collision frequency of plasmas in an unambiguous way. Molmud ${ }^{22}$ has suggested the use of complex effective collision frequencies in the Lorentz model, while Whitmer and Hermann ${ }^{25}$ have defined both an effective collision frequency and an effective plasma frequency; other possibilities are also available. For example, Shkarofsky ${ }^{26}$ has used g and h functions. For the purpose of analyzing the experimental data presented in this paper, the prescription suggested by Molmud ${ }^{23}$ appears to be the most convenient, even though the method becomes less accurate as the neutral gas pressure is increased. $Q$ for Helium is reasonably independent of electron velocity, which means that the collision frequency $\nu_{m}$ varies directly as the velocity $v$; the mean free path of the electrons $L=v / \nu$ is, hence, a constant.

Molmud ${ }^{23}$ has shown that for a constant $Q$ the expressions for $B$ and $D$ as given by equations $(6 b)$ and $(6 c)$ reduce to the following form:

$$
B=\frac{1}{\omega} \frac{4 x^{1 / 2}}{3 \sqrt{\pi}} 1=x-x^{2} e^{x} E_{i}(-x)
$$




$$
D=\frac{1}{\omega} \frac{4 x}{3 \sqrt{\pi}}\left[\left(\frac{1}{2}-x\right) \pi^{1 / 2}+\pi x^{3 / 2} e^{x}(1-\phi(V x))\right]
$$

where

$$
\begin{aligned}
& x=\frac{4 \omega^{2}}{\pi \nu_{m}^{2}} ; \nu_{m}=2 \rho Q /(\pi \beta)^{1 / 2} \\
& -E_{i}(-x)=\int_{x}^{\infty} \frac{e^{-t}}{t} d t
\end{aligned}
$$

and

$$
\phi(z)=\frac{2}{\sqrt{\pi}} \int_{0}^{z} \exp \left(-t^{2}\right) d t
$$

where $E_{i}(-x)$ and $\phi(z)$ are the Exponential Integral and Error Integral, respectively. On equating the conductivity expressions for the two models given by equations (5) and (6), the complex effective collision frequency for the Lorentzian case necessary for equalizing the two conductivities is given by

$$
\nu_{e}=\nu_{e R}+j \nu_{e I}
$$

ductivities is given by

When $\nu_{\mathrm{m}} / \omega<1$

$$
\nu_{\mathrm{eR}}=\frac{4}{3} \nu_{\mathrm{m}}\left(1-0.22 \nu_{\mathrm{m}}^{2} / \omega^{2}\right)
$$

and

$$
\nu_{e I}=0.18\left(\nu_{m}^{2} / \omega^{2}\right)\left[1+2.14 \nu_{m}^{2} / \omega^{2}\right]
$$

When $\nu_{m} / \omega>1$,

$$
\nu_{e R}=\left(3 \pi \nu_{m} / 8\right)\left(1+0.28 \omega^{2} / \nu_{m}^{2}\right)
$$

and

$$
\nu_{\mathrm{eI}}=0.18 \omega\left(1-7.5 \omega^{2} / \nu_{\mathrm{m}}^{2}\right)
$$


when $\nu_{\mathrm{m}}$ in the above two equations is strictly defined as $\nu_{\mathrm{m}}=\int \rho Q(\mathrm{v}) \mathrm{Fv} \mathrm{dv}$. For Helium with a constant $Q$, this reduces to the form

$$
\nu_{m}=2 \rho Q\left(2 \mathrm{kT}_{\mathrm{e}} / \pi \mathrm{m}\right)^{1 / 2}
$$

Equations (14a) and (14b) indicate that when the imaginary part of the complex effective frequency is small $\left(\nu_{e I} \ll \nu_{e R}\right)$, the collision frequency for momentum transfer $\nu_{m}$ can be obtained from the effective collision frequency $\nu_{e}$ of the Lorentzian model using the relation

$$
\nu_{e}=\frac{4}{3} \nu_{m}\left(1-0.22 \nu_{m}^{2} / \omega^{2}\right) \quad \text { when } \nu_{m} / \omega<1
$$

and

$$
\nu_{\mathrm{e}}=\left(3 \pi \nu_{\mathrm{m}} / 8\right)\left(1+0.28 \omega^{2} / \nu_{\mathrm{m}}^{2}\right) \quad \text { when } \nu_{\mathrm{m}} / \omega>1
$$

In order to obtain the relative magnitudes of $\nu_{e I}$ and $\nu_{e R}$, the complex effective collision frequency for Helium was calculated for various neutral gas pressures used in the experimental investigation; in these calculations the electron temperature $T_{e}$ was 6.5 electron volts and the signal frequency $\omega / 2 \pi$ was 450 MegaHertz. The results are shown in Figure 1 . It is seen from this figure that the assumption $\nu_{e I} \ll \nu_{e R}$ is valid only at pressures below $500 \mu \mathrm{Hg}$. At higher pressures $\nu_{\text {eI }}$ increases rapidly in value and, thus, there is no simple method of obtaining $\nu_{m}$ from the measured effective collision frequency $\nu_{e}$ In the high pressure limit it may become necessary to use the method of Whitmer and Hermann ${ }^{25}$ and prescribe both an equivalent electron density and collision frequency to relate the Lorentzian and kinetic theory conductivity expressions. 


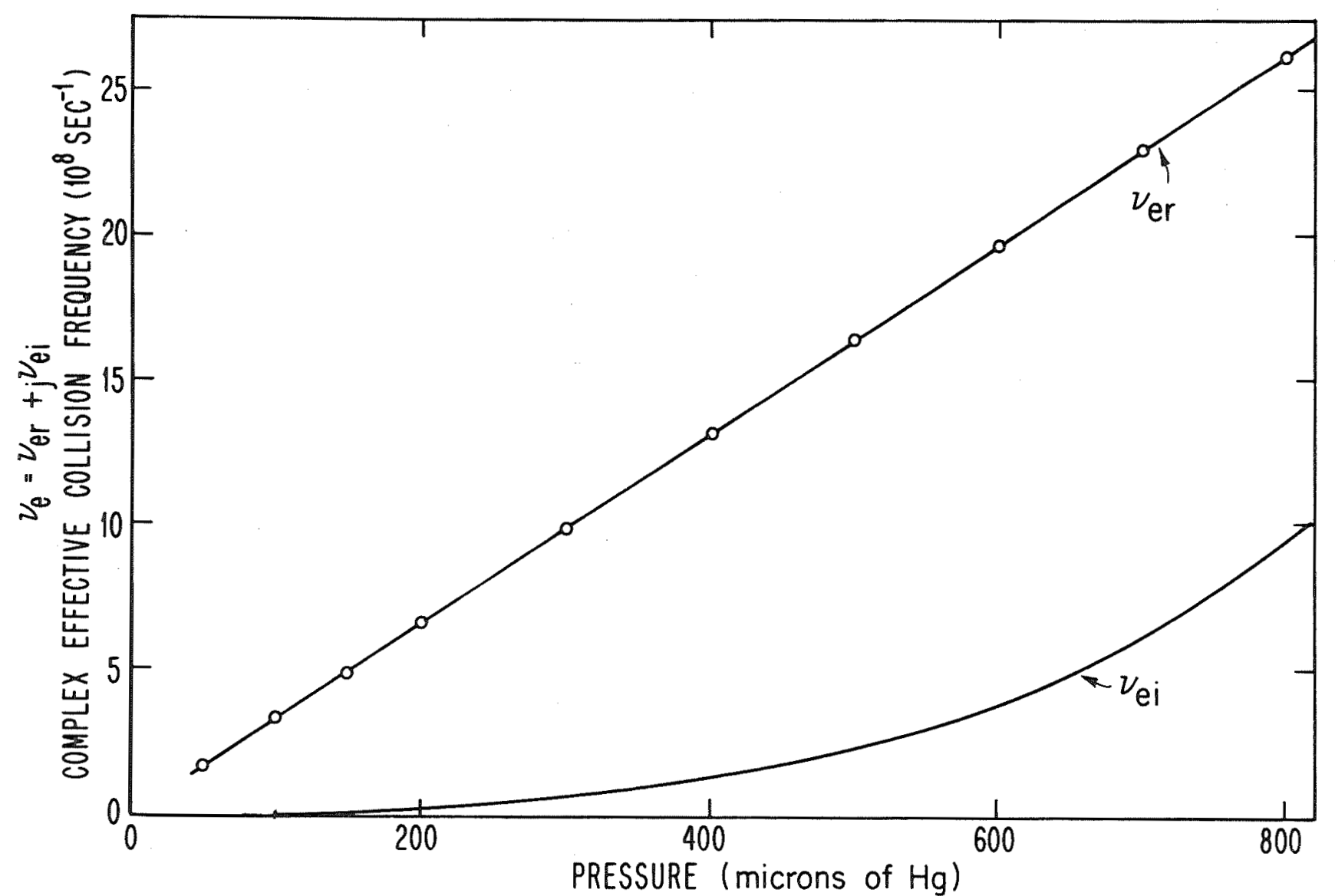

COMPLEX EFFECTIVE COLLISION FREQUENCY REQUIRED FOR EQUATING LORENTZIAN AND KINETIC THEORY MODELS FOR PLASMA CONDUCTIVITY. (ELECTRON TEMPERATURE $T_{e}=6.5 \mathrm{eV}$ COLLISION CROSS SECTION $=4.5 \mathrm{~A}^{2}$ FREQUENCY $=450 \mathrm{MHZ}$ ) 
4. Experimental Procedure and Apparatus.

In the experimental investigations to be described in the following sections, the collision frequency $\nu_{m}$ is measured by using the following procedure: a) precision impedance measurements are made on the antenna at 11 frequencies over a 2 to 1 range, typically 300 to $600 \mathrm{MHz}$, which includes the electron plasma frequency. It is preferable to have the plasma frequency fall nearly in the middle of this range (the approximate plasma frequency may be found by using some independent method such as the Langmuir probe). b) Using a 4 th degree interpolation polynomial the frequencies at which the maximum values of the resistance, $R$ meas, and magnitude of the impedance, $|\mathrm{Z}|^{\text {meas }}$, occur are determined as well as the values themselves. c) Using the frequency of $|Z|_{\max }^{\text {meas }}$ as the plasma

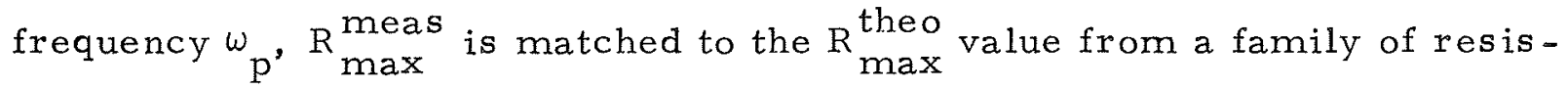
tance curves derived from King's theory (eq. 1) with the same plasma frequency, but with the collision frequency as the parameter. ${ }^{*}$ d) The value of the collision frequency for momentum transfer $\nu_{m}$ is then obtained from either equation (16a) or (16b) provided $\nu_{e I} \ll \nu_{e R}$ e) Lastly, to obtain an estimate of the accuracy of the results, the value of $\nu_{m}$ obtained in this manner is compared with the theoretical results for $\nu_{\mathrm{m}}$ calculated using the collision cross-section data of Golden and Bandel. 3

This scheme has been formalized and a computer program written to process the measured data and return the plasma frequency and effective collision frequency. At high collision frequencies the assumption that $|\mathrm{Z}|$ max occurs at the electron plasma frequency is not accurate. But, for the laboratory plasma measured using the above technique, the error involved in the determination of $\nu_{e}$ and $\omega_{p}$ is only a few percent $(\approx 5 \%)$. Thus, it is questionable whether one should use a more sophisticated curve fitting procedure. 
A block diagram of the experimental apparatus is shown in Figure 2. The expeximental investigations were made in a hot-cathode, Helium d.c. discharge tube, $14 \mathrm{cms}$ in diameter and $38 \mathrm{cms}$ in length. The antenna was a copper rod, $4 \mathrm{mms}$ in diameter and $3.5 \mathrm{cms}$ in length. Since the length of the antenna is very short compared to the wavelength of the r.f. signal frequency used in the experiment, the radiation field is negligible and the plasma discharge around the antenna has a significant effect only on the reactive near field of the antenna. Hence, the finite size of the plasma container does not seriously compromise the 'infinite' plasma assumption made in the theoretical analysis. This was investigated experimentally by using plasma columns of larger and larger diameter, until the size of the plasma column had a negligible effect on the antenna impedance. The electron density profile in the plasma column is ambipolar diffusion controlled with a radial variation of the type $n(r)=n(0) J_{0}(2.404 r / a)$ where $a$ is the radius of the discharge tube and $n(0)$ is the electron density at the axis. Since the antenna was placed along the axis of the positive column, it is evident that when the radius of the discharge column is sufficiently large, the fields of the antenna see essentially a homogeneous plasma. The antenna was connected to the inner conductor of a vacuum-tight precision coaxial connector; this eliminates large junction effects near the driving point of the antenna. The coaxial connector was mounted at the center of a copper disc which also served as the anode of the discharge tube. The electron density and electron temperature of the plasma were determined by a planar Langmuir probe. The Langmuir probe V-I characteristics were scanned electronically at the rate of once every two seconds with a semi-log plot displayed on a cathode ray tube. This rate is slow enough to 


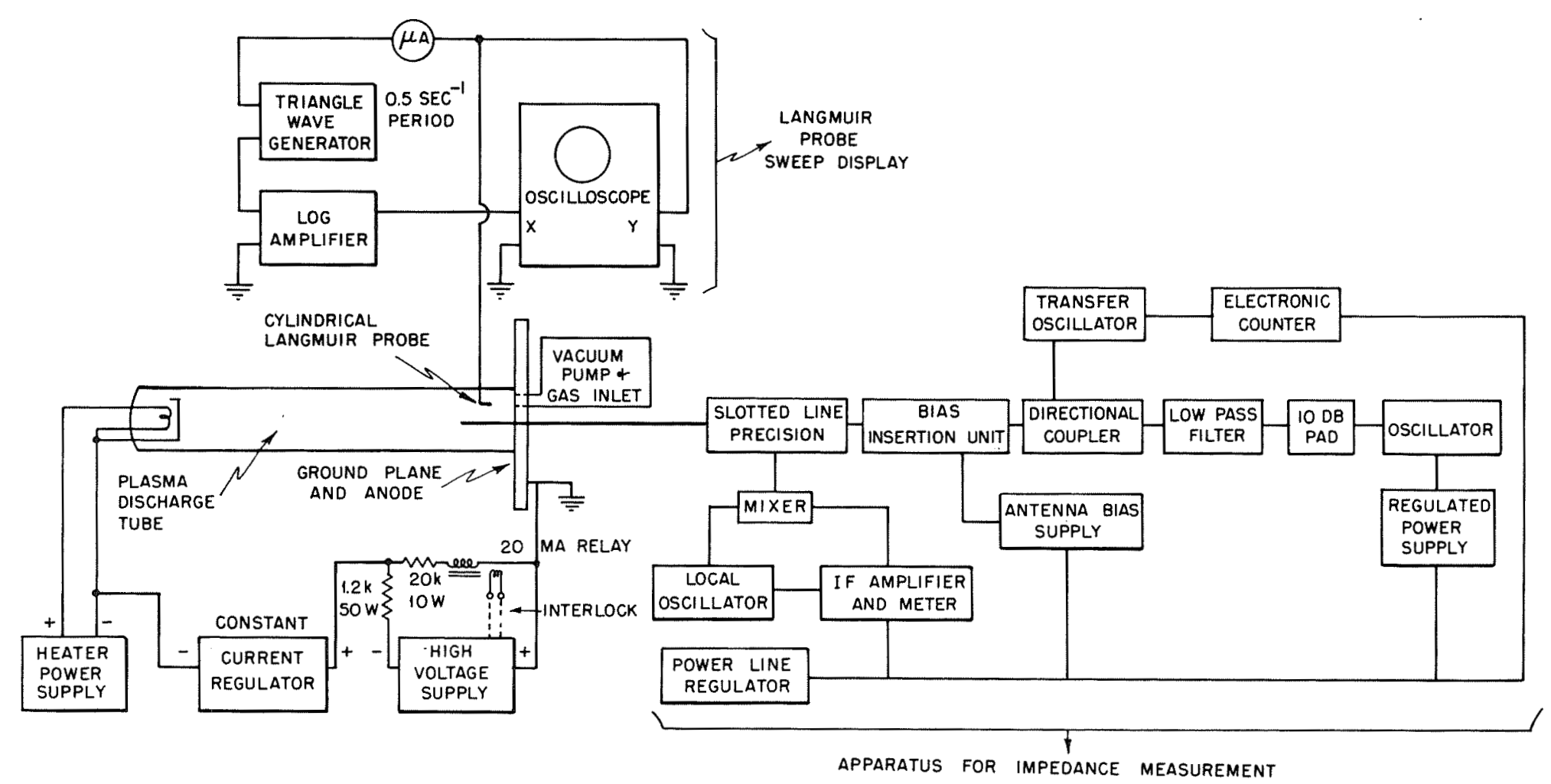

FIG. 2 BLOCK DIAGRAM OF EXPERIMENTAL APPARATUS 
make any hysterisis in the system negligible but fast enough to exclude drift effects in the plasma discharge. The semi-log plot allows a direct reading of the electron temperature. This was facilitated by a calibrated overlay placed on the CRT face. Since the electron temperature is important both for calculating collision frequencies as well as estimating electroacoustic effects, great care was exercised in making these measurements. The value of the electron temperature measured by this technique was compared with the theoretical values calculated from the cPR $\mathrm{vs} \mathrm{T}_{\mathrm{e}} / \mathrm{V}_{\mathbf{i}}$ characteristics for Helium (see Ref, 26, Fig, 14-11). The ionization potential for Helium $V_{i}=24.46$ volts and $c=3.9 \times 10^{-3}, \quad P$ is the neutral gas pressure in mms of $\mathrm{Hg}$ and $\mathrm{R}$ is the radius of the discharge column in cms. The results are shown in Figure 3; they indicate good agreement between theory and experiment.

The electron-neutral collision frequency for momentum transfer $\nu_{m}=2.66 \times 10^{22} \times \sqrt{T_{e}} Q_{m} P$ was determined from the collision crosssections for Helium measured by Golden and Bandel. ${ }^{3}$ In the above equation $\mathrm{T}_{\mathrm{e}}$ is the electron temperature of the plasma in degrees Kelvin, $\mathrm{P}$ is the neutral gas pressure in $\mathrm{mms}$ of $\mathrm{Hg}$, and $\mathrm{Q}_{\mathrm{m}}$ is the electron-neutral collision cross-section for momentum transfer in $\mathrm{cms}^{2}$. Since the plasma is weakly ionized it is assumed that the electron-ion collisions are negligible.

\section{Comparison of Experimental Results with Theoretical Results of King} and Applications to Plasma Diagnostics.

The antenna impedance was measured as a function of $\omega_{p} / \omega$ by varying the signal frequency in the vicinity of the plasma frequency. The plasma frequency during the experiment was stabilized by using a constant 


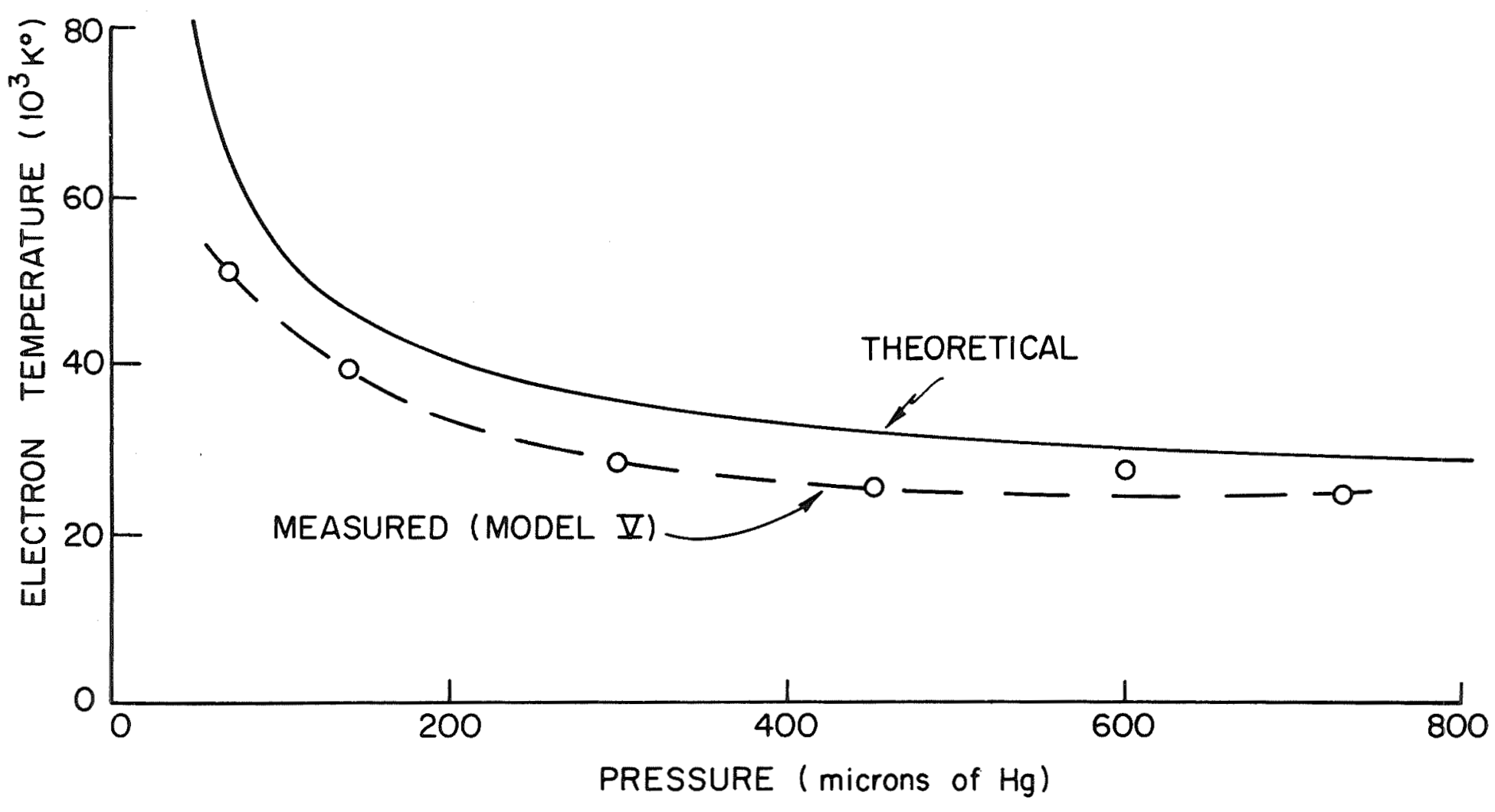

FIG. 3 COMPARISON OF MEASURED ELECTRON TEMPERATURE WITH THEORETICAL VALUES CALCULATED FROM THE CPR Vs. Te/Vi CURVE FOR A HELIUM POSITIVE COLUMN (FIG. 14-l/ OF S.C. BROWN'S BASIC DATA OF PLASMA PHYSICS, M.I.T. PRESS 1959) 
current regulator in series with the d.c. power supply to the discharge tube. The impedance measurements were made at various neutral gas pressures and the results were compared with the theoretical values of the impedance obtained from King's theory (Equation 1). The values of $\nu_{e}$ and $\omega_{p}$ were obtained by a curve-fitting technique described earlier. The comparison between theoretical and experimental results for measurements made at neutral gas pressures of 140,300 , and 750 microns of $\mathrm{Hg}$ are shown in Figure 4. The theoretical curves were obtained by setting $\omega_{\mathrm{p}} / \omega=1$ at $Z_{\max }^{\text {measured }}=\sqrt{R^{2}+x^{2}}$ and $R_{\max }^{\text {theoretical }}=R_{\max }^{\text {measured }}$. It can be seen from this figure that there is good agreement between theory and experiment for values of $\omega_{\mathrm{p}} / \omega$ between 0.8 and 1.6 . This demonstrates that the shape of the theoretical curve is correct and that King's theory gives a good description of the impedance behavior of the antenna in the vicinity of the plasma frequency of a collision-dominated plasma. Having obtained the experimental value for $\nu_{e}$ in this manner, $\nu_{m}$ was then obtained from equation 16. In Figure 5 the experimental values for $\nu_{e}$ are compared with the theoretical values obtained from the collision cross - section data of Golden and Bandel. ${ }^{3}$ It is seen that there is fairly good agreement, although the experimental values appear to be somewhat larger than that predicted by theory. This increase is probably due to a) errors in measuring neutral gas pressures due to calibration errors in the thermocouple gauge, b) impurities in the gas system which can increase $\nu_{e^{\prime}}$ and c) non-collisional damping phenomena which have not been taken into account in either of the theories. Noncollisional damping effects have also been noticed by Waletzko and Bekefi $^{27}$ and by Crawford and $\mathrm{Harp}^{28}$ in impedance measurements made 


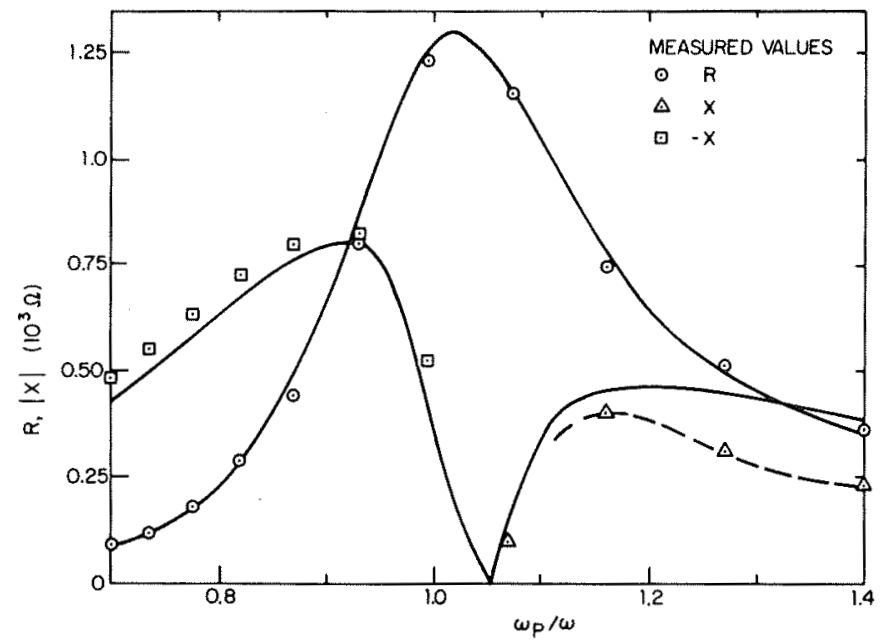

4a PRESSURE $=140$ MICRONS; MEASURED PLASMA

FREQUENCY $=418$ MHZ; MEASURED COLLISION FREQUENCY :

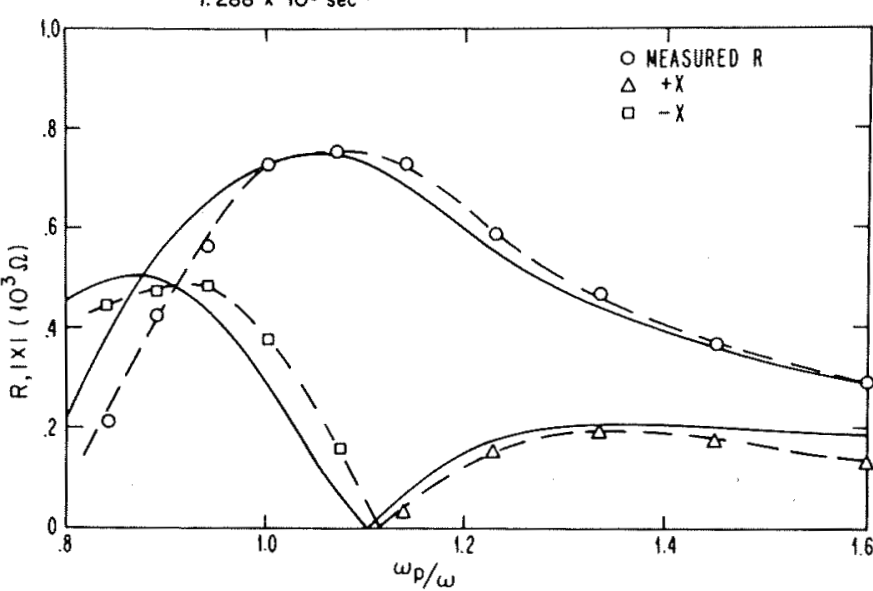

46 PRESSURE $=300$ MICRONS; MEASURED PLASMA FREQUENCY = $477 \mathrm{MHz}$; MEASURED COLLISION FREOUENCY $=12.79 \times 10^{\circ} \mathrm{sec}^{-1}$

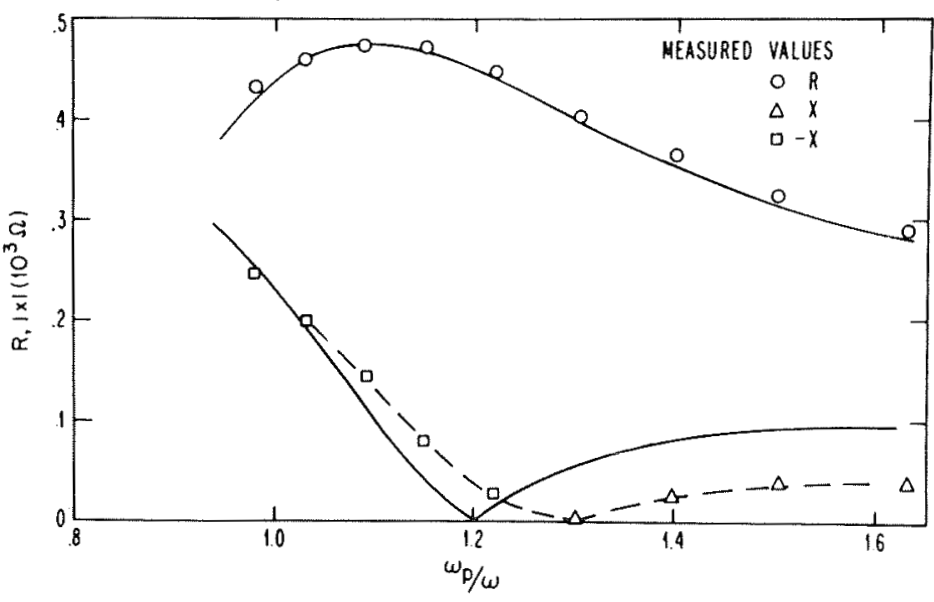

$4 \mathrm{C}$ PRESSURE $=730 \mu ;$ MEASURED PLASA FREQUENCY $=587 \mathrm{MHz}$ MEASURED COLLISION FREQUENCY $=21.19 \times 10^{8} \mathrm{sec}^{-1}$

FIG 4 COMPARISON OF THEORETICAL AND EXPERIMENTAL RESULTS FOR THE IMPEDANCE OF A SHORT DIPOLE ANTENNA IN A PLASMA IGAS-HELIUM, $h=3.49 \times 10^{-2}$ METERS; $a=2.13 \times 10^{-3}$ METERS 


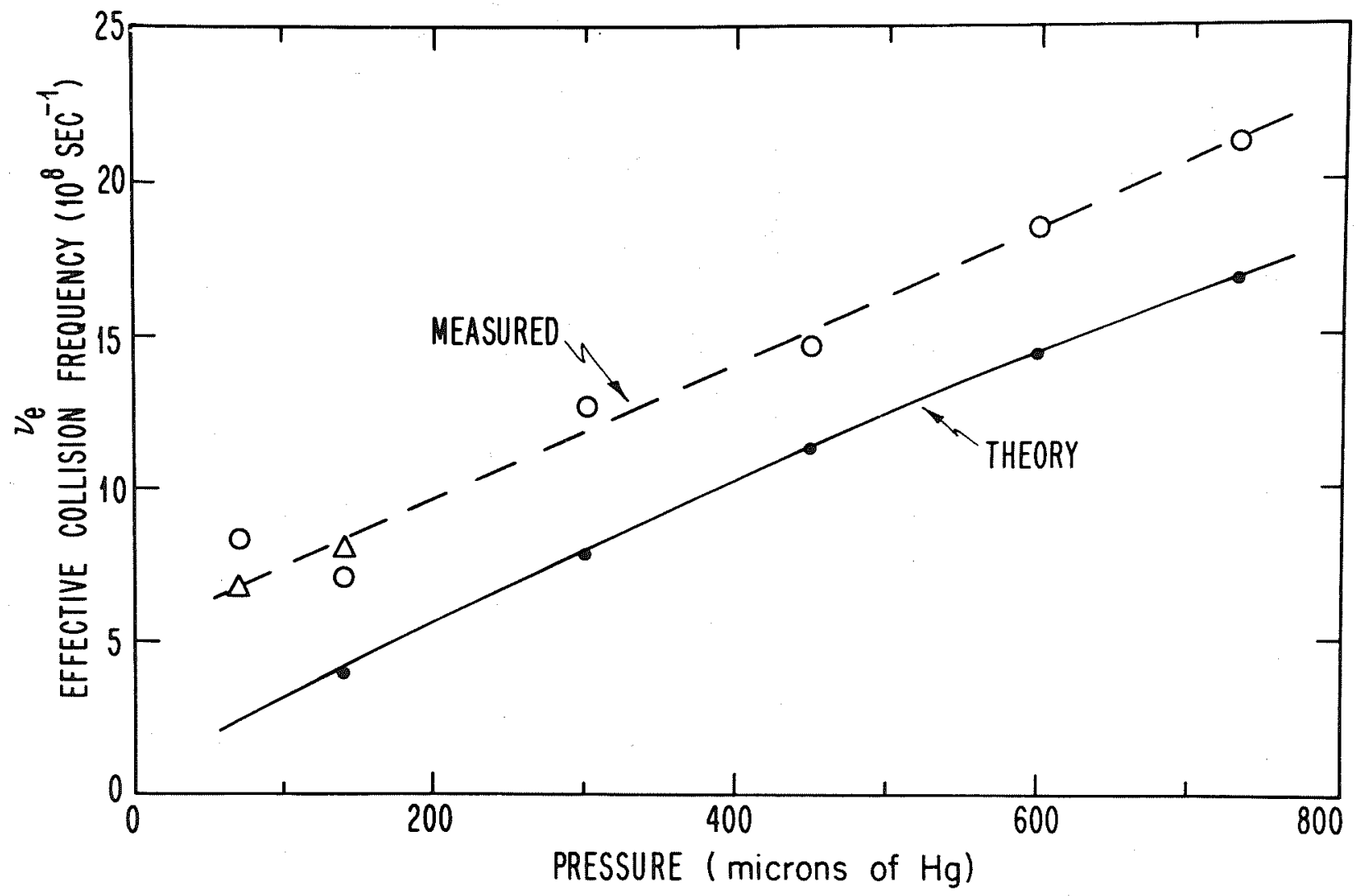

COMPARISON BETWEEN MEASURED AND THEORETICAL VALUES FOR THE EFFECTIVE ELECTRONNEUTRAL COLLISION FREQUENCY FOR MOMENTUM TRANSFER.

FIG. 5 
with a spherical probe immersed in an isotropic plasma. The results shown in Figure 5, however, indicate that, in general, reasonable values for the collision frequency can be obtained by using the antenna as a diagnostic probe in a collision dominated plasma. The value of the electron density obtained from the 'curve-fitting' procedure shown in Figure 4 was compared with independent density measurements made with a planar Langmuir probe. The results are compared in Table 1 and show once again that the antenna can be used for making reliable measurements of the electron density.

6. Effect of Electron Temperature and Ion-Sheath Effects on Antenna Impedance.

It is obvious from the previous discussion that the experimental results are in good agreement with King's theory ${ }^{1}$ based on the cold plasma model. For warm plasmas, having significant electron temperature, Balmain's theory (Equation 4) indicates that an additional resistance term is necessary to account for the electroacoustic radiation by the antenna. If resistance term $R_{p}$ due to this plasma mode is $s$ mall compared to the resistance $\mathrm{R}_{\text {em }}$ due to the electromagnetic mode, the cold plasma assumption is still valid insofar as the diagnostic measurements are concerned. If, however, $R_{p}$ and $R_{\text {em }}$ are comparable, significant errors are likely to occur if the cold plasma theory is used for making collision frequency measurements. In order to estimate the relative magnitudes of $R_{e m}$ and $R_{p}$ calculations were made using equation (4) for typical plasma parameters occurring in the experiments just described. The results are shown in Figure 6 ; the vallues of $\nu_{e}, T_{e}, \omega$ and 
TABLE 1

Comparison of Plasma Frequency Determined by a Planar Langmuir Probe and from R.F. Impedance Measurements using a Short Cylindrical Antenna

Neutral Gas Pressure of Helium (microns)
Discharge Current (milliamps)
Plasma Frequency Short Antenna $(\mathrm{MHz})$

450

418

477

510

630

587
Plasma Frequency

Langmuir Probe $(\mathrm{MHz})$

404

420

420

458

543

458 


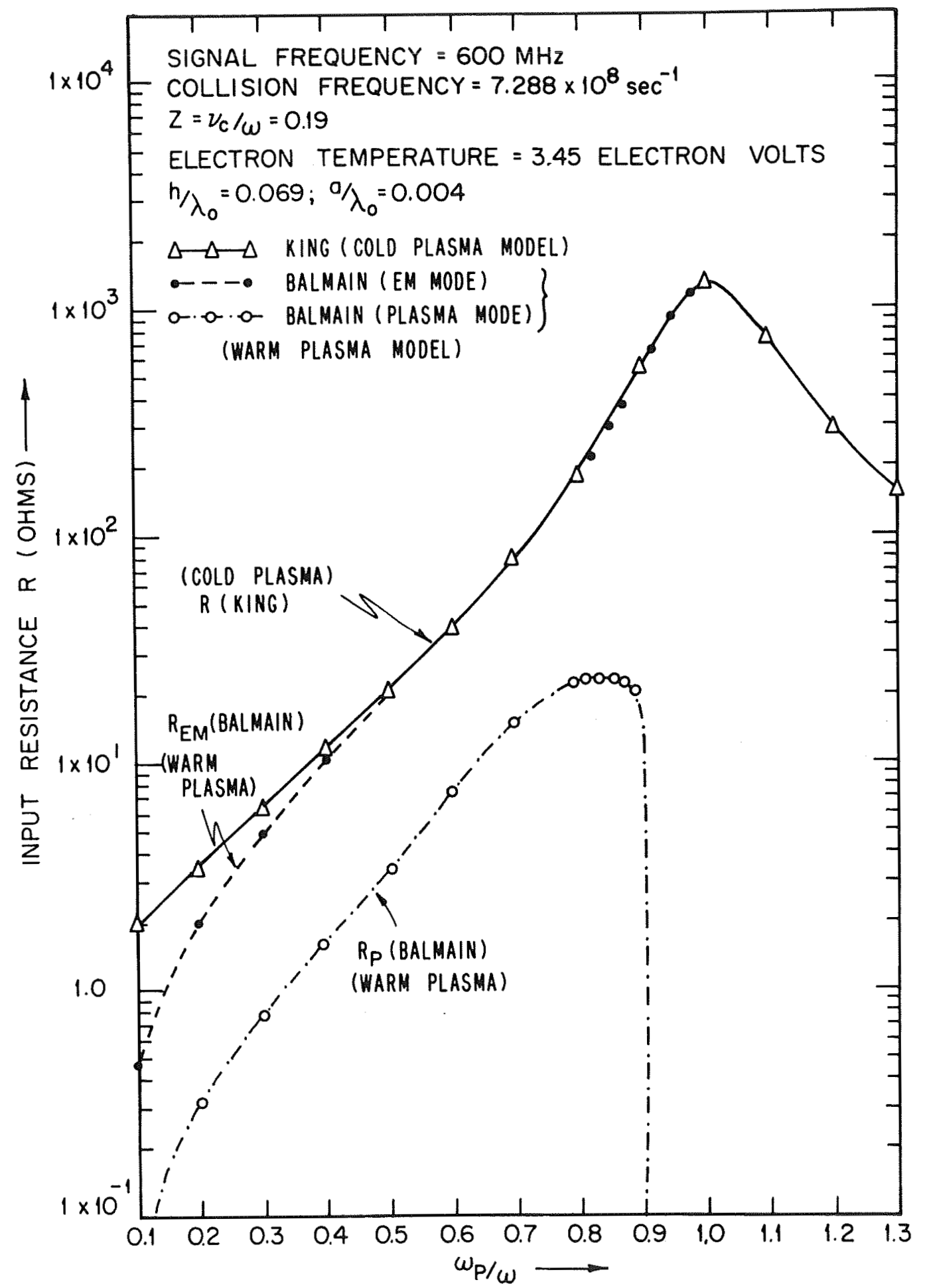

FIG. 6 EFFECT OF ELECTRON TEMPERATURE ON INPUT RESISTANCE OF THE ANTENNA.-COMPARISON OF COLD AND WARM PLASMA MODELS. 
the antenna dimensions used in these calculations are similar to those occurring in the impedance measurements shown in Figure $4 a$. It is seen from this figure that $R_{p}$ reaches its maximum value of 22.78 ohms at $\omega_{\mathrm{p}} / \omega=0.84$ where the corresponding $R_{\mathrm{em}}=270 \mathrm{ohms}$. Hence, since the maximum value of $R_{p} / R_{e m}=0.084$, the electroacoustic mode makes a negligible contribution to the input resistance of the antenna when the collision frequency is the dominant loss mechanism. It is also interesting to note that $R_{p}$ falls off sharply to zero as the plasma frequency is approached. The resistance $R$ calculated from King's theory (Equation 1 ) is also plotted in Figure 6. It is seen that $R_{\text {em }}$ obtained from Balmain's theory agrees closely with $R$ of King's for $\omega_{\mathrm{p}} / \omega$ between 0.5 and 1.0. For $\omega_{\mathrm{p}} / \omega<0.4, \mathrm{R}$ em drops off sharply to zero and differs significantly from King's theory; this discrepancy is due to the quasi-static approximations made by Balmain in his analysis, as a consequence of which the radiation resistance is neglected.

In Figure 7 the theoretical values for $R_{p}$, computed for various electron temperatures ranging from 3 to 50 electron volts, have been plotted as a function of $\omega_{\mathrm{p}} / \omega . \mathrm{R}$ em has also been plotted in the same figure for comparison. The signal frequency $\omega / 2 \pi$ was $600 \mathrm{MHz}$ and the effective collision frequency $\nu_{e}$ was $0.16 w$ in these calculations. The plasma is assumed to be an idealized Maxwellian gas, so that the effective collision frequency is independent of the electron temperature. This figure indicates that for collision frequencies of this magnitude, the maximum value of $R_{p} / R_{e m}=$ 0.185 even for electron temperatures $\mathrm{T}_{\mathrm{e}}$ as high as 10 electron volts. For $R_{p}$ to become comparable to $R_{e m}$, the electron temperature must correspond to 50 electron volts; these are abnormally high electron temperatures which are rarely encountered in laboratory plasmas where a typical $T_{e}$ is of the 


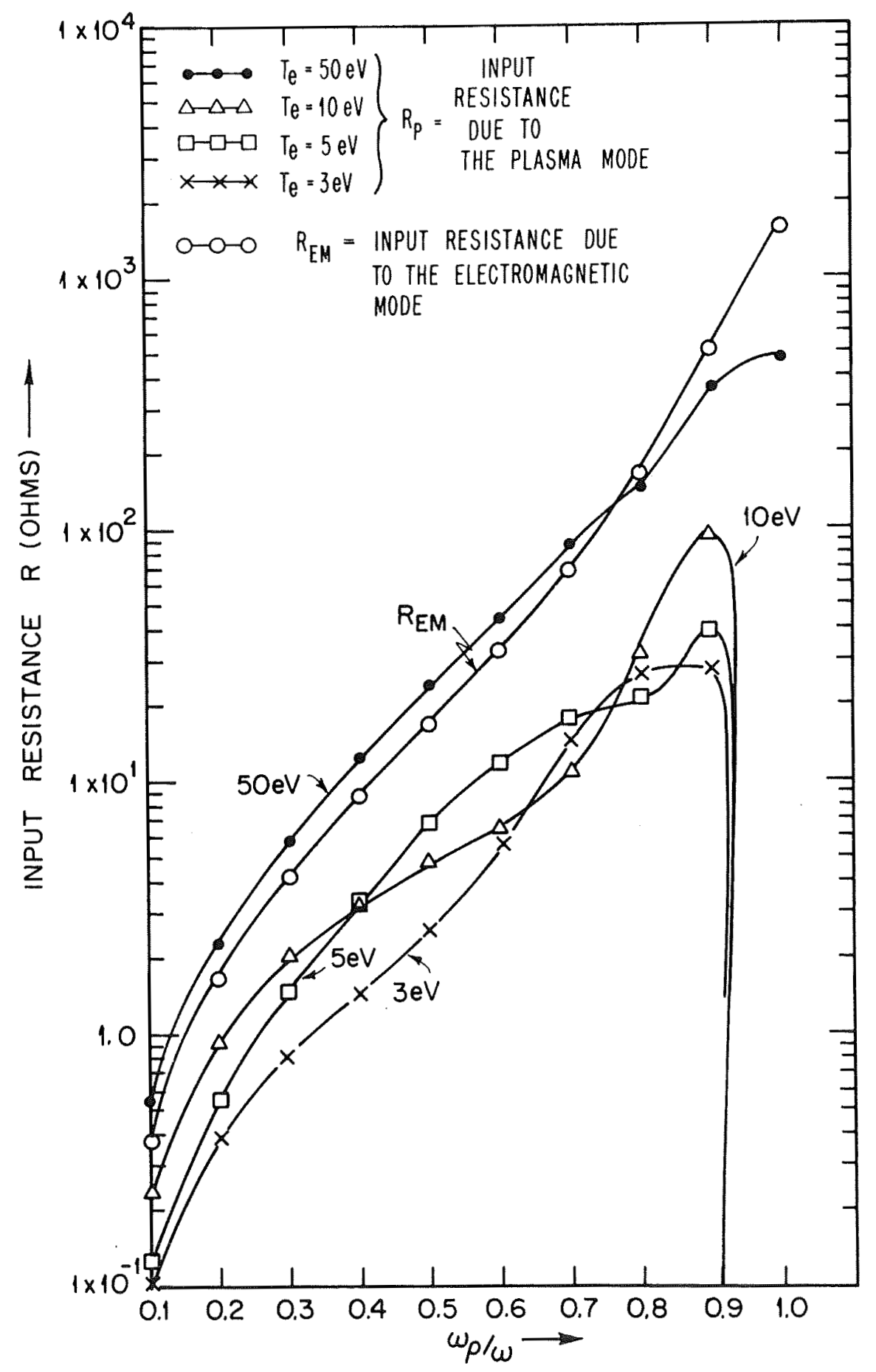

FIG. 7 INPUT RESISTANCE OF ANTENNA FOR VARIOUS ELECTRON TEMPERATURES. 
order of a few electron volts. The electron temperatures in the ionosphere vary from 300 to $1000^{\circ} \mathrm{K}(0.025$ to 0.09 electron volts) with the highest collision frequencies occurring in the D-region $(60-80$ kilometers) where $\nu_{\mathrm{e}}$ varies from 13 to 0.2 megacycles. ${ }^{7}$ The results shown in Figure 7 indicate that for most laboratory plasmas, the collisions rather than the electron temperature play a dominant role in determining the input resistance in the vicinity of the plasma frequency. In Figure 8 the input resistance has been calculated for various $\nu_{e} / \omega$ from King's cold plasma theory. These results show that collisional losses as small as $\nu_{\mathrm{e}} / \omega=0.001$ can result in a significant peaking of the input resistance in the neighborhood of the plasma frequency. For very high collision frequencies $\left(\nu_{e} / \omega=1,0\right)$ the resistance varies in a smooth manner near $\omega_{\mathrm{p}}$. In order to apply this impedance probe technique for plasma diagnostics, an error analysis was performed by considering various values of $\nu_{e}$ and $T_{e}$. The range where the probe can be used for determining $\nu_{e}$ within $10 \%$ was determined and is shown in Figure 9. Carlin and Mittra 29 have shown that the power in the electroacoustic mode drops off sharply when the dimensions of the antenna are large compared to the electroacoustic wavelength. For an electron temperature of 5.2 electron volts $\left(6 \times 10^{4}\right.$ Kelvin), the electroacoustic wavelength is typically of the order of $10^{-2} \mathrm{cms}$ for a signal frequency of 1 Gigattertz. Hence, by using long antennas it is possible to reduce the maximum value of $R_{p}$ even further, so that the probe can be used for diagnosing even plasmas having low collisional losses.

The effect of the ion-sheath on the impedance of the bare antenna. was investigated by applying various $d . c$ bias voltages to the antenna to vary the sheath thickness. This was generally found to have a negligible 


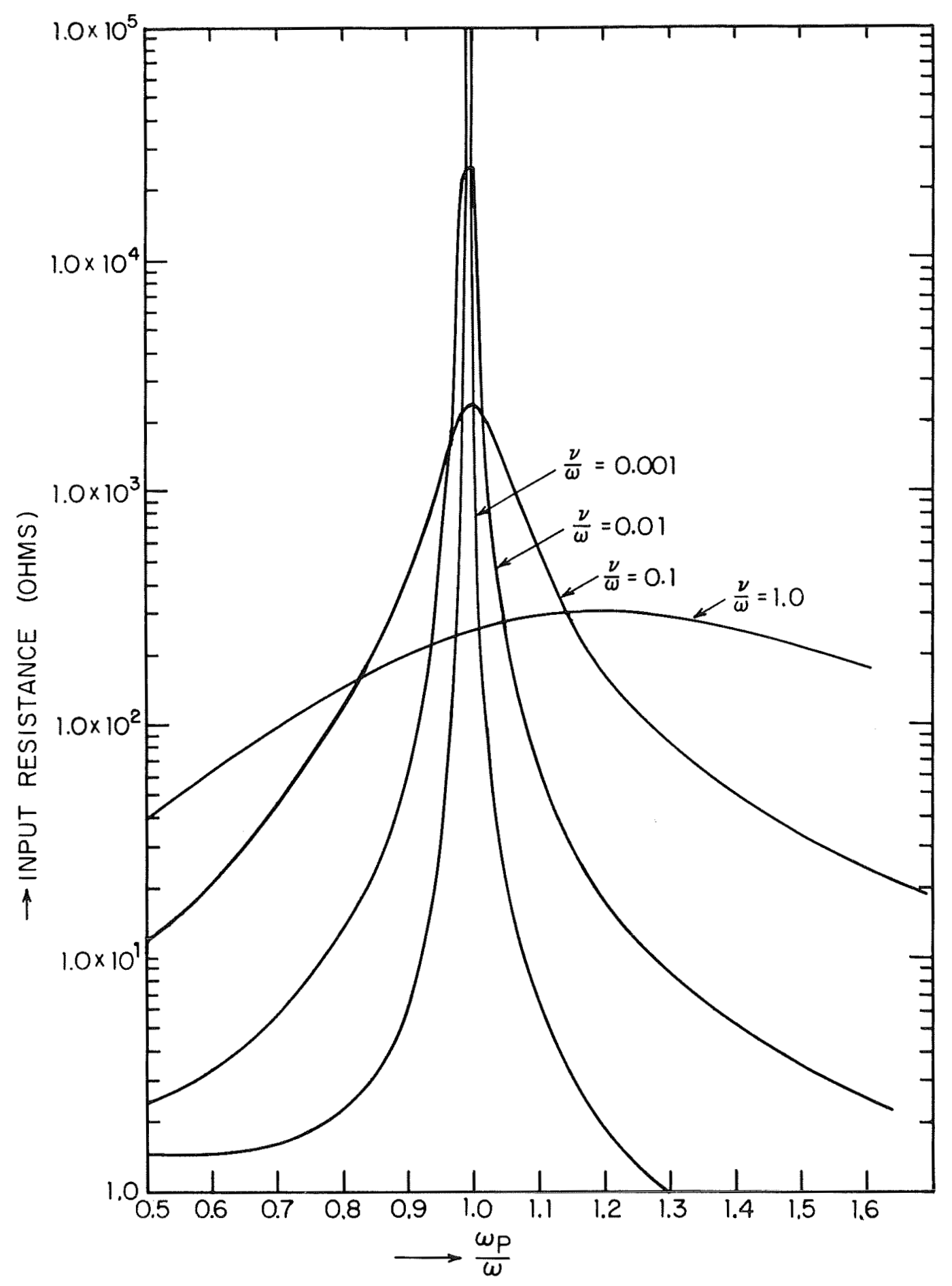

VARIATION IN INPUT RESISTANCE OF A SHORT ANTENNA AS A FUNCTION OF COLLISION FREQUENCY, $h / \lambda=0.0698 ; a / \lambda=0.0042$ FIGURE 8 


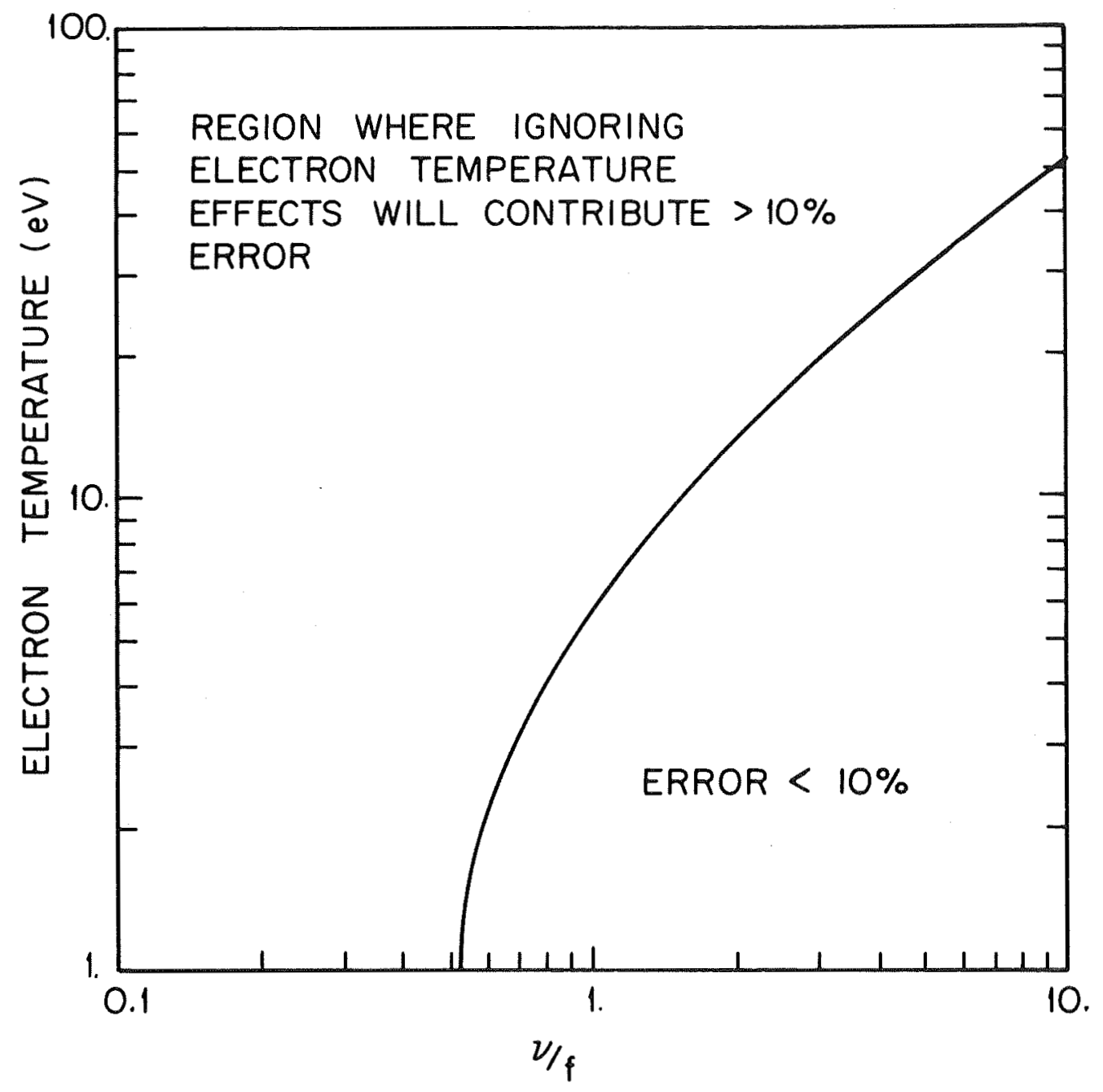

FIG. 9 DOMAIN OF VALIDITY FOR COLLISION FREQUENCY MEASUREMENT USING THE IMPEDANCE PROBE TECHINIQUE. (DETERMINED FOR $f=600 \mathrm{MHZ}_{Z}$ AND $\omega_{\mathrm{p}} / \omega=0.9$ USING BALMAIN'S FORMULA AS A REFERENCE.) 
effect on the antiresonant impedance behavior of the antenna near $\omega_{\mathrm{p}}$. This is probably due to the antenna radius being much larger than the Debye wavelength of the plasma used in these investigations. For an electron density $\mathrm{n}=2 \times 10^{9}$ electrons $/ \mathrm{cm}^{3}$ and an electron temperature $\mathrm{T}_{\mathrm{e}}=5$ electron volts, the Debye wavelength is approximately $10^{-2} \mathrm{cms}$, as compared to the antenna radius of $0.2 \mathrm{cms}$. Experiments by Waletzko and Bekefi ${ }^{27}$ and Harp and Crawford ${ }^{28}$ on a spherical probe indicate that ion-sheath effects become noticeable only when the antenna dimensions are of the same order of magnitude as the Debye length. Investigations by Mlodnosky and Garriott ${ }^{11}$ indicate that the effects of an ion-sheath on the antenna admittance are noticeable only at frequencies well below the plasma frequency of a low density lossless plasma. Another probable reason is that when the collision frequencies become significant, the 'resonance' conductance peak gets damped rapidly. Buckley ${ }^{30}$ has shown that for a spherical resonance probe, the width of the conductance peak is proportional to $0.1 \omega_{\mathrm{p}}+1.6 \nu_{\mathrm{e}}$. Since the $\nu_{\mathrm{e}}$ in our experiments were quite large, it is quite likely that the resonance effects were too heavily damped to be noticeable.

\section{Conclusions}

Our investigations show that 1) an electrically short cylindrical antenna can be used for diagnosing the electron density and collision fre. quency of the plasma, 2) near the plasma frequency the influence of collisional losses on the antenna resistance is similar to that obtained from including electroacoustic wave effects, 3) the resistance contribution due to the electroacoustic mode is negligible when the collision frequency is 
high, so that the 'cold' plasma theory is applicable for interpreting experimental data, 4) the velocity dependence of the collision frequency can be satisfactorily accounted for by introducing a fictitious complex effective collision frequency, and 5) the effect of the ion-sheath on the antenna impedance near $\omega_{\mathrm{p}}$ is small when the electron density and collision frequency are large. 


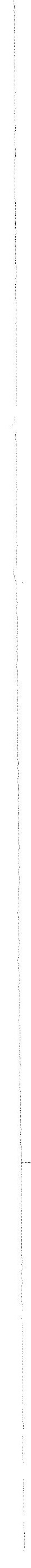




\section{ACKNOWLEDGMENT}

The authors thank Prof。 R. W. P. King for his advice and encouragement and Drs. A. D. Wunsch and A. E. Sanderson for several helpful discussions during the course of this investigation. The assistance rendered by $\mathrm{Mr}$. A. Cajolet and Mr。D. Macmillan in the construction of the experimental apparatus is also gratefully acknowledged. This research was supported by the National Aeronautics and Space Administration Agency under Grant NGR 22-007-056。 


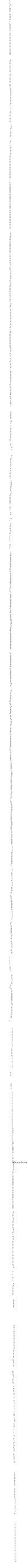




\section{BIBLIOGR A PHY}

1. R. W. P. King, C. W. Harrison, Jr。 and D. H. Denton, "The Electrically Short Antenna as a Probe for Measuring Free Electron Densities and Collision Frequencies in an Ionized Region", J. Res. Natl. Bur. Stds, Vol. 65D, No.4, 371-384 (1961).

2. K. G. Balmain, "Impedance of a Short Dipole in a Compressible Plasma'", Radio Science, Vol.69D, No.4, 559-566 (1965).

3. D. E. Golden and H. W. Bandel, "Absolute Total Electron-Helium Atom Scattering Cross Sections for Low Electron Energies", Physical R eview, Vol。138, A14-A21 (April 1965).

4. R. H. Huddlestone and S. L. Leonard, Plasma Diagnostic Techniques, Academic Press, Chapter 4, 113-199 (1965).

5. F. W. Crawford and R. S. Harp, "The Resonance Probe - A Tool for Ionospheric and Space Research", J. Geophys。Res, Vol.70, 587-596 (1965).

6. M. A. Heald and C. B. Wharton, Plasma Diagnostics with Microwaves, John Wiley \& Sons, Chapter 6, 192-241(1965).

7. J. A. Kane, "Arctic Measurements of Electron Collision Frequencies in the D-region of the Ionosphere", Journal of Geophysical Research, Vol. 64, 133-139 (Feb。1959).

8. J. M. Anderson and L. Goldstein, "Interaction of Electromagnetic Waves of $R$ adio-Frequency in Isothermal Plasmas: Collision Cross Section of Helium Atoms and Ions for Electrons", Physical Review, Vol. 100, 1037-1046 (1955).

9. O. C. Haycock, K. D. Baker and J. C. Ulwick, "Experiences with the Impedance Probe on Satellites", Proc. IEEE, Vol. 52, 1029-1033 (1964).

10. J. E. Jackson and J. A. Kane, "Measurement of Ionospheric Electron Densities Using an $r$. f. Probe Technique", J. Geophys. Res., Vol.64, 1074 (1959a).

11. R. F. Mlodnosky and O. K. Garriott, "The VLF Admittance of a Dipole in the Lower Ionosphere", Proc。 of the International Conference on the Ionosphere, Phys. Soc。(London), 484-491 (1962).

12. B. Bhat and B. Rama Rao, "Experimental Investigations on the Impedance Behavior of a Short, Cylindrical Antenna in a Lossy Magnetoplasma", Cruft Lab. Scientific Report No。2, Harvard University (Jan. 1969). 
13. I, P. Shkarofsky, T. W. Johnston and M。 P. Bachynski, The Particle Kinetics of Plasmas, Addison Wesley Pub. Co., Chapter 4, $118-149(1966)$.

14. M. P. Bachynski, "Sources in Unbounded Plasmas", (paper presented at the 15th General Assembly of URSI, Munich, Germany) RCA Victor Research Report No. $7=801-50$ (July 1966).

15. J. Galejs, "The Insulated Cylindrical Antenna Immersed in a Compressible Plasma", Sylvania Research Report No. 560 (Sept. 1968).

16. A. D. Wunsch, "The Finite Tubular Antenna in a Warm Plasma", R adio Science, Vol。3, No, 9, 901-920 (1968).

17. S. H. Lin and K. K. Mei, "Numerical Solution of Dipole Radiation in a Compressible Plasma", IEEE Trans。on Antennas and Propagation, Vol. AP-16, 235-242 (1968).

18. K. R. Cook and B. C. Edgar, "Current Distribution and Impedance of a Cylindrical Antenna in an Isotropic, Compressible Plasma", Radio Science, Vol。1 (New Series), No, 1, 13-19 (1966).

19. H. H. Kuehl, "Computations of the Resistance of a Short Antenna in a Warm Plasma", Radio Science, Vol。 2 (New Series), No. 1, 73-76 (1967).

20. W. P. Allis, Handbuch der Physik, Springer-Verlag, Berlin, Vol, 21, 392 (1956).

21. H. Margenau, "Conductivity of Plasmas to Microwaves", Phys. Rev., Vol, 109, 6-9 (Jan. 1958).

22. P. Molmud, "Langevin Equation and the $a_{0} c_{0}$ Conductivity of NonMaxwellian Plasmas", Physical R eview, Vol.114,29-32 (Apri1, 1959).

23. H. Margenau and D. Stillinger, "Microwave Conductivity of Slightly Ionized Air", J.Appl。 Phys。, Vol。30, No, 9, 1385-1387 (Sept, 1959).

24. J. A. Kane, "Re-evaluation of Ionospheric Electron Densities and Collision Frequencies Derived from Rocket Measurements of Refractive Index and Attenuation", J. Atmos. Terrest. Phys。, Vol. 23, 338 (1961).

25. R. F. Whitmer and G. F. Hermann, "Effects of a Velocity-Dependent Collision Frequency on Wave-Plasma Interactions", Phys of Fluids, Vol。9, No, 4, 768-773 (1966).

26. S. C. Brown, Basic Data of Plasma Physics, MIT Press, Figure 14. 11, 291 1959).

27. J. A. Waletzko and G. Bekefi, "R.F. Admittance Measurements of a Slotted-Sphere Antenna Immersed in a Plasma", Radio Science, Vol. 2 (New Series), No. 5, 489-493 (1967). 
28. R. S. Harp and F. W. Crawford, "Characteristics of the Plasma Resonance Probe", J.Appl。Phys., Vol。35, 3436 (1964).

29. J. Carlin and R. Mittra, "Acoustic Waves and Their Effect on Antenna Impedance", Can.J. Phys. Vol.45, 1251-1269 (March 1967),

30. R. Buckley, "The Response of a Spherical Plasma Probe to Alternating Potentials", Journal of Plasma Physics, Vol. 1, Part2, $171-191(1967)$. 